\title{
Diabetes: can we stop the time bomb?
}

\author{
M Fisher
}

Heart 2003;89(Suppl II):ii28-ii30

Type 2 diabetes is increasing in prevalence and it is estimated that three million individuals in the UK will have the disease by 2010. People with diabetes have a high risk of cardiovascular disease-coronary heart disease is more prevalent, more extensive, and more diffuse. For all cardiovascular syndromes, the mortality is virtually doubled in patients with diabetes. Large clinical trials have provided clear evidence of the reduction in cardiovascular risk that can be achieved by treating hyperglycaemia as well as traditional risk factors, such as hypertension and hyperlipidaemia. The challenge now is for all health care professionals to implement the treatment guidelines.

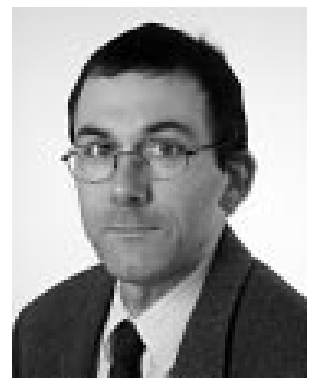

$T$ ype 2 diabetes currently affects almost two million people over the age of 16 in the UK, ${ }^{1}$ although only half are diagnosed. By 2010 it is estimated that nearly three million individuals will have the disease in the UK and up to 300 million people worldwide. Type 2 diabetes occurs more frequently in the elderly ${ }^{2}$ and the obese. Since the UK elderly population is increasing, there will be an increasing number of people predisposed to the disease. Likewise, increasing levels of obesity and sedentary behaviour, especially in young people, are also contributing to the escalation of type 2 diabetes.

Given the high cardiovascular risk associated with diabetes, the disease might be redefined as "a state of premature cardiovascular death which is associated with chronic hyperglycaemia and may also be associated with blindness and renal failure". Many patients do not live long enough to go blind or for their kidneys to fail.

The findings of a recent diabetes mortality study demonstrate that diabetes is a serious cardiovascular condition. A population based cohort of 4842 people with diabetes living within South Tees was identified and followed from

Table 1 Interventions that can reduce cardiovascular risk in diabetes

\begin{tabular}{|c|c|}
\hline Hypoglycaemic treatment & $\begin{array}{l}\text { Metformin (UKPDS) } \\
\text { Sulfonylureas/insulin }\end{array}$ \\
\hline Risk factor reduction & $\begin{array}{l}\text { Hypertension } \\
\text { Hyperlipidaemia }\end{array}$ \\
\hline Antiplatelet treatment & $\begin{array}{l}\text { Aspirin } \\
\text { Clopidogrel }\end{array}$ \\
\hline ACE inhibition & Ramipril \\
\hline Lifestyle? & \\
\hline
\end{tabular}

Dr Miles Fisher, Glasgow Royal Infirmary, Glasgow G31 2ER, UK; miles.fisher@ northglasgow.scot.nhs.uk
ACE, angiotensin converting enzyme; UKPDS, UK Prospective Diabetes Study.
January 1994 to December 1999. ${ }^{3}$ Causes of death were obtained from death certificates and mortality rates were compared with the non-diabetic population of the same area for the same period. There were 1205 deaths $(24.9 \%)$ in the study population over the six years of the study. For type 2 diabetes, mortality from cardiovascular causes was significantly increased for both sexes. Echoing Framingham data, but in a modern British setting, the study showed that for diabetic men aged 40-59 years, relative to non-diabetic men, there was at least doubling of all cause mortality; cardiovascular deaths and ischaemic heart disease deaths both increased fivefold. For women aged 40-59 years, the figures were even more striking: all cause mortality relative to nondiabetic women of the same age increased threefold, cardiovascular deaths increased fivefold, and ischaemic heart disease deaths increased ninefold.

Coronary heart disease in people with diabetes has been investigated in epidemiological studies, postmortem studies, and using electrocardiography and angiography. These studies show that the disease is more prevalent, more extensive (it tends to be triple vessel disease), and more diffuse (multiple segments of disease within one artery) in patients with diabetes compared with nondiabetic subjects. It is also more silent, with many patients having no symptoms and therefore not presenting for treatment. Coronary heart disease is also more lethal in patients with diabetes-for all cardiovascular syndromes, the mortality is virtually doubled.

\section{EVIDENCE FOR REDUCING CARDIOVASCULAR RISK}

On the positive side, clinical trials have provided clear evidence on action that can be taken to reduce cardiovascular risk in patients with diabetes (table 1). Cardiovascular benefit is provided by treating hyperglycaemia as well as by treating traditional risk factors, such as hypertension and hyperlipidaemia. Lifestyle interventions are, however, still unproven as there are no cardiovascular outcome data from randomised controlled trials.

For treating hyperglycaemia, the treatment of choice in overweight patients is metformin. In the

Abbreviations: $A C E$, angiotensin converting enzyme; ALLHAT, Antihypertensive and Lipid Lowering Treatment to Prevent Heart Attack Trial; CAPRIE, Clopidogrel Versus Aspirin in Patients at Risk of Ischaemic Events; CARE, Cholesterol and Recurrent Events; DAIS, Diabetes Atherosclerosis Intervention Study; HOPE, Heart Outcomes Prevention Evaluation; LIPID, Long term Intervention with Pravastatin in Ischaemic Disease; LIPS, Lescol Intervention Prevention Study; UKPDS, UK Prospective Diabetes Study; VA-HIT, Veterans Affairs High Density Lipoprotein Cholesterol Intervention Trial; 4S, Scandinavian Simvastatin Survival Study 


\begin{tabular}{|c|c|c|c|c|}
\hline \multirow[t]{2}{*}{ Overweight patients } & \multicolumn{4}{|c|}{$\operatorname{RR}(95 \% \mathrm{Cl})$} \\
\hline & RR & $p^{0.2}$ & 1 & 1 \\
\hline $\begin{array}{c}\text { Any diabetes related end point } \\
\text { Metformin }\end{array}$ & 0.68 & 0.0023 & 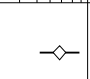 & \\
\hline $\begin{array}{c}\text { Diabetes related deaths } \\
\text { Metformin }\end{array}$ & 0.58 & 0.017 & 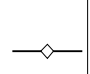 & \\
\hline $\begin{array}{l}\text { All cause mortality } \\
\text { Metformin }\end{array}$ & 0.64 & 0.011 & 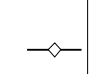 & \\
\hline $\begin{array}{r}\text { Myocardial infarction } \\
\text { Metformin }\end{array}$ & 0.61 & 0.01 & 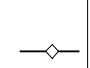 & \\
\hline
\end{tabular}

Figure 1 Effect of metformin in overweight patients. Adapted from UKPDS,${ }^{4}$ with permission.

UK Prospective Diabetes Study (UKPDS), ${ }^{4}$ intensive blood glucose control with metformin had benefit over conventional treatment in terms of both small vessel and large vessel disease. Metformin was associated with reduction in diabetes related deaths and all cause mortality, with the benefit being greater than would have been predicted on the basis of glycaemic control ( fig l).

Data on treatment of hypertension in diabetes come from studies carried out exclusively in patients with diabetes, such as UKPDS, and from studies in wider patient populations that included large cohorts of patients with diabetes. There is a substantial evidence base for treatment of hypertension in diabetes and in selected subgroups. The recently published Antihypertensive and Lipid-Lowering Treatment to Prevent Heart Attack Trial (ALLHAT) included a large diabetic cohort. ${ }^{5}$ Several groups of antihypertensive drugs have proven benefit in patients with diabetes, including angiotensin converting enzyme (ACE) inhibitors, angiotensin II receptor antagonists, $\beta$ blockers, diuretics, and some of the calcium channel blockers. The $\alpha$ blocker doxazosin is not recommended as a first line treatment for hypertension in patients with diabetes ${ }^{6}$ but is a useful add-on agent for blood pressure control.

There is also a large evidence base on the use of statins to treat hyperlipidaemia and reduce cardiovascular risk in patients with diabetes. In primary prevention, the heart protection study ${ }^{7}$ showed the benefit of simvastatin, while the Anglo Scandinavian Cardiac Outcomes Trial (ASCOT) is yet to be published, but the lipid lowering component has been prematurely stopped because of benefit from statin treatment. For secondary prevention, subgroup analysis from the major trials showed a beneficial effect with simvastatin in the Scandinavian Simvastatin Survival Study $(4 S)^{8}$ and the Heart Protection Study, ${ }^{7}$ with pravastatin in the Cholesterol And Recurrent Events (CARE) study ${ }^{9}$ and the Long term Intervention with Pravastatin in Ischaemic Disease (LIPID) study, ${ }^{10}$ and with fluvastatin in the Lescol Intervention Prevention Study (LIPS). ${ }^{11}$ Gemfibrozil was of proven benefit in the Veterans Affairs High Density Lipoprotein Cholesterol Intervention Trial (VA-HIT). ${ }^{12}$ Fenofibrate produced positive results in the Diabetes Atherosclerosis Intervention Study (DAIS), ${ }^{13}$ although benefit is still uncertain as this trial used a surrogate outcome (angiographic progression of coronary artery disease). The hyperlipidaemia studies show that patients with diabetes have the same relative risk reduction as non-diabetic subjects; however, because the event rate is so much higher, more lives are saved with the intervention in patients with diabetes.

The effect of antiplatelet agents on vascular events in diabetes has been assessed in an updated meta-analysis of nine trials. ${ }^{14}$ These drugs were shown to provide statistically significant benefit although this was limited, with only a 7\% proportional reduction in serious vascular events. The effect of the newer antiplatelet agent clopidogrel has also been studied

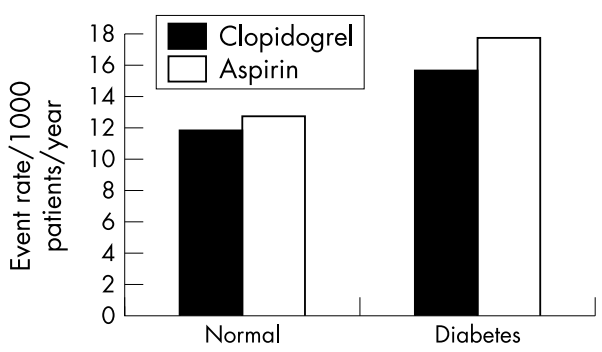

Figure 2 Data from the CAPRIE study showing higher rate of cardiovascular events (myocardial infarction, stroke, vascular death. and rehospitalisation for ischaemic events) in patients with diabetes. Adapted from Bhatt et al, ${ }^{15}$ with permission.

in patients with diabetes. The Clopidogrel versus Aspirin in Patients at Risk of Ischaemic Events (CAPRIE) study included a significant number of patients with diabetes. ${ }^{15}$ Data from this trial are shown in fig 2. Although this includes a "soft" end point (hospitalisation for ischaemic events), it again highlights the fact that patients with diabetes have a much higher rate of cardiovascular events than non-diabetics and that achieving the same relative benefit with an intervention (in this case either aspirin or clopidogrel) will therefore save more lives.

In the Heart Outcomes Prevention Evaluation (HOPE) study, ${ }^{16}$ about a third of patients had diabetes. Use of the ACE inhibitor ramipril led to a $25 \%$ reduction in the primary outcome (myocardial infarction, stroke or cardiovascular death) compared with placebo. There is controversy over the interpretation of the study in terms of whether the observed effect is related simply to blood pressure lowering or to some other effect of ACE inhibition. For a clinician, however, it can be argued that a treatment that reduces cardiovascular risk by $25 \%$ should be prescribed irrespective of exactly how it is working.

\section{CONCLUSION}

People with diabetes are probably the highest risk group for cardiovascular disease. Several guidelines are available on reducing cardiovascular risk in people with diabetes, based on a large amount of evidence from clinical trials. The challenge for health care professionals in both primary and secondary care is to implement the treatment guidelines to try to reduce the cardiovascular burden in people with diabetes. Polypharmacy is an inevitable consequence of the need to intervene on several different risk factors and this can lead to problems with compliance and adherence.

\section{REFERENCES}

1 Amos AF, McCarty DJ, Zimmet P. The rising global burden of diabetes and its complications: estimates and projections to the year 2010. Diabet Med 1997:14(suppl 5):S1-85.

2 Budd SC, Gatling W, Currell I, et al. The incidence of non-insulin dependent diabetes mellitus in the community: extrapolation to the UK suggests over 95000 new cases per year [abstract]. Diabet Med 1998; suppl 2:S11, abstract A30.

3 Roper NA, Bilous RW, Kelly WF, et al. Cause-specific mortality in a population with diabetes: South Tees diabetes mortality study. Diabetes Care 2002;25:43-8.

4 UK Prospective Diabetes Study (UKPDS) Group. Effect of intensive blood-glucose control with metformin on complications in overweight patients with type 2 diabetes (UKPDS 34). Lancet 1998-352:854-65.

5 The ALLHAT Officers and Coordinators for the ALLHAT Cooperative Research Group. Major outcomes in high-risk hypertensive patients randomized to angiotensin-converting enzyme inhibitor or calcium channel blocker vs diuretic: the antihypertensive and lipid lowering treatment to prevent heart attack trial (ALLHAT). JAMA 2002;288:2981-97.

6 The ALLHAT Officers and Coordinators for the ALLHAT Collaborative Research Group. Major cardiovascular events in hypertensive patients randomized to doxazosin vs chlorthalidone: the antihypertensive and lipid-lowering treatment to prevent heart attack trial (ALLHAT). JAMA 2000;283:1967-75. 
7 Heart Protection Study Collaborative Group. MRC/BHF heart protection study of cholesterol lowering with simvastatin in 20536 high-risk individuals: a randomised placebo-controlled trial. Lancet 2002;360:7-22.

8 Scandinavian Simvastatin Survival Study Group. Randomised trial of cholesterol lowering in 4444 patients with coronary heart disease: the

Scandinavian simvastatin survival study (4S). Lancet 1994;344:1383-9.

9 Sacks FM, Pfeffer MA, Moye LA, et al. The effect of pravastatin on coronary events after myocardial infarction in patients with average cholesterol levels. N Engl J Med 1996:335:1001-9.

10 The Long-Term Intervention with Pravastatin in Ischaemic Disease (LIPID) Study Group. Prevention of cardiovascular events and death with pravastatin in patients with coronary heart disease and a broad range of initial cholesterol levels. N Engl J Med 1998;339:1349-57.

11 Serruys PW, de Feyter P, Macaya C, et al. Fluvastatin for prevention of cardiac events following successful first percutaneous coronary

intervention: a randomized controlled trial. JAMA 2002;287:3215-22.

12 Rubins HB, Robins SJ, Collins D, et al for the Veterans Affairs High-Density Lipoprotein Cholesterol Intervention Trial Study Group.
Gemfibrozil for the secondary prevention of coronary heart disease in men with low levels of high-density lipoprotein cholesterol. N Engl J Med 1999;341:410-8.

13 Diabetes Atherosclerosis Intervention Study Investigators. Effect of fenofibrate on progression of coronary-artery disease in type 2 diabetes: the diabetes atherosclerosis intervention study, a randomised study. Lancet 2001;357:905-10.

14 Antithrombotic Trialists' Collaboration. Collaborative meta-analysis of randomised trials of antiplatelet therapy for prevention of death, myocardial infarction, and stroke in high risk patients. BM 2002;324:71-86.

15 Bhatt DL, Marso SP, Hirsch AT, et al. Amplified benefit of clopidogre versus aspirin in patients with diabetes mellitus. Am J Cardiol 2002;90:625-7.

16 Heart Outcomes Prevention Evaluation (HOPE) Study Investigators. Effects of ramipril on cardiovascular and microvascular outcomes in people with diabetes mellitus: results of the HOPE study and MICRO-HOPE substudy. Lancet 2000;355:253-9. 